\title{
Self-nanoemulsifying drug delivery systems ameliorate the oral delivery of silymarin in rats with Roux-en-Y gastric bypass surgery
}

This article was published in the following Dove Press journal:

International Journal of Nanomedicine

25 March 2015

Number of times this article has been viewed

\author{
Chun-Han Chen ${ }^{1,2}$ \\ Cheng-Chih Chang' \\ Tsung-Hsien Shih ${ }^{2}$ \\ Ibrahim A Aljuffali ${ }^{3}$ \\ Ta-Sen Yeh ${ }^{4,5}$ \\ jia-You Fang ${ }^{6-8}$ \\ 'Division of General Surgery, \\ Department of Surgery, Chang Gung \\ Memorial Hospital, Chiayi, ${ }^{2}$ Graduate \\ Institute of Clinical Medical Sciences, \\ College of Medicine, Chang Gung \\ University, Kweishan, Taoyuan, Taiwan; \\ ${ }^{3}$ Department of Pharmaceutics, \\ College of Pharmacy, King Saud \\ University, Riyadh, Saudi Arabia; \\ ${ }^{4}$ Department of Surgery, Chang Gung \\ Memorial Hospital, ${ }^{5} \mathrm{School}$ of \\ Medicine, College of Medicine, \\ ${ }^{6}$ Pharmaceutics Laboratory, Graduate \\ Institute of Natural Products, \\ ${ }^{7}$ Chinese Herbal Medicine Research \\ Team, Healthy Aging Research Center, \\ Chang Gung University, ${ }^{8}$ Research \\ Center for Industry of Human \\ Ecology, Chang Gung University of \\ Science and Technology, Kweishan, \\ Taoyuan, Taiwan
}

Correspondence: Ta-Sen Yeh Department of Surgery, Chang Gung Memorial Hospital, 5 Fuxing Street, Kweishan, Taoyuan 333, Taiwan $\mathrm{Tel}+8863328 \quad 1200$ ext 2745

Email tsy471027@cgmh.org.tw

Jia-You Fang

Pharmaceutics Laboratory, Graduate Institute of Natural Products, Chang Gung University, 259 Wen-Hwa Ist Road, Kweishan, Taoyuan 333, Taiwan Tel +88632 II 8800

Fax +88632118236

Email fajy@mail.cgu.edu.tw
Abstract: Roux-en-Y gastric bypass (RYGB) is a popular surgery to reduce the body weight of obese patients. Although food intake is restricted by RYGB, drug absorption is also decreased. The purpose of this study was to develop novel self-nanoemulsifying drug delivery systems (SNEDDS) for enhancing the oral delivery of silymarin, which has poor water solubility. The SNEDDS were characterized by size, zeta potential, droplet number, and morphology. A technique of RYGB was performed in Sprague-Dawley rats. SNEDDS were administered at a silymarin dose of $600 \mathrm{mg} / \mathrm{kg}$ in normal and RYGB rats for comparison with silymarin aqueous suspension and polyethylene glycol (PEG) 400 solution. Plasma silibinin, the main active ingredient in silymarin, was chosen for estimating the pharmacokinetic parameters. SNEDDS diluted in simulated gastric fluid exhibited a droplet size of $190 \mathrm{~nm}$ with a spherical shape. The nanocarriers promoted silibinin availability via oral ingestion in RYGB rats by 2.5 -fold and 1.5-fold compared to the suspension and PEG 400 solution, respectively. A significant doublepeak concentration of silibinin was detected for RYGB rats receiving SNEDDS. Fluorescence imaging showed a deeper and broader penetration of Nile red, the fluorescence dye, into the gastrointestinal mucosa from SNEDDS than from PEG 400 solution. Histological examination showed that SNEDDS caused more minor inflammation at the gastrointestinal membrane as compared with that caused by PEG 400 solution, indicating a shielding of direct silymarin contact with the mucosa by the nanodroplets. SNEDDS generally showed low-level or negligible irritation in the gastrointestinal tract. Silymarin-loaded SNEDDS were successfully developed to improve the dissolution, permeability, and oral bioavailability of silymarin. To the best of our knowledge, this is the first investigation reporting the usefulness of SNEDDS for improving drug malabsorption elicited by gastric bypass surgery.

Keywords: self-nanoemulsifying drug delivery system, Roux-en-Y gastric bypass, obesity, oral delivery, silymarin

\section{Introduction}

Epidemiological investigation has shown an increased prevalence of obesity worldwide. ${ }^{1}$ Obesity can result in poor health, reduction in quality of life, and a high risk of depression. A $20 \%$ increase in body weight is associated with a $20 \%$ increase in risk of death. ${ }^{2}$ In order to reverse this trend, many strategies are being attempted to treat morbid obesity. Bariatric surgery is demonstrated to be the most effective choice for weight reduction in obese patients. ${ }^{3}$ Among the bariatric surgery techniques, Roux-en-Y gastric bypass (RYGB) is one of the most popular, with the advantages of maintaining long-term weight loss and decreasing morbidity. In RYGB, a small part of the stomach is used to create a gastric pouch. The stomach pouch is then connected to the middle of the jejunum by circumventing the rest of the stomach, duodenum, and 
proximal jejunum. ${ }^{4}$ However, RYGB dramatically decreases the surface area for absorption by bypassing significantly long portions of the gastrointestinal (GI) tract, leading to malabsorption of nutrients/drugs and diminishing drug solubility in the GI tract. ${ }^{5,6}$ Moreover, oral administration is the preferred pathway for drug delivery due to its convenience and likelihood of patient compliance. The development of drug delivery systems for RYGB patients is important for improving the currently insufficient bioavailability.

Self-nanoemulsifying drug delivery systems (SNEDDS) have gained growing attention for solving the problem of poor oral absorption of drugs, especially permeants with low water solubility. ${ }^{7}$ Digestive motility in the GI tract enables the stirring required to form nanoemulsions. The advantages of SNEDDS include ease of large-scale preparation, thermodynamic stability, lack of need for organic solvents, and improved oral drug bioavailability. ${ }^{8}$ In the present work, an attempt was made to develop SNEDDS as a way of enhancing the solubility and permeability of drugs given via the oral route in rats undergoing RYGB. The rat model can closely reproduce bariatric surgery in humans. ${ }^{9}$

Obesity is often accompanied by liver abnormalities known as nonalcoholic fatty liver disease. The prevalence of steatosis, a marker of nonalcoholic fatty liver disease, is $85 \%$ for extremely obese individuals. ${ }^{10}$ Silymarin is an effective treatment for reducing hepatic steatosis in patients with nonalcoholic fatty liver disease. ${ }^{11}$ Silymarin is isolated from milk thistle, which is a mixture of flavonolignans, with silibinin as the most abundant and active ingredient. This drug is commonly used to treat chronic liver disease due to its hepatoprotective activity. ${ }^{12}$ The therapeutic effect of silymarin was discounted for its poor water solubility and low GI permeability. The oral bioavailability of silymarin is only $23 \%-47 \% .{ }^{13}$ Because of this problem, silymarin was used as the model drug in this study. SNEDDS loaded with silymarin were physicochemically examined for droplet size and number, zeta potential, and morphology. A comparative bioavailability study was carried out in normal and RYGB rats using SNEDDS, silymarin suspension, and silymarin polyethylene glycol (PEG) 400 solution. The depth of penetration of the nanocarriers into the GI membrane was monitored by fluorescence imaging.

\section{Materials and methods \\ Materials}

Silymarin, silibinin, sulfatase, $\beta$-glucuronidase, narigenin, PEG 400, Cremophor ${ }^{\circledR}$ RH40, Tween 20, and Nile red were purchased from Sigma-Aldrich (St Louis, MO, USA).
The lot number for silymarin was BCBJ0393V. The percentage of silibinin in silymarin was $45.0 \%$ according to the certificate of analysis provided by the vendor. DAPI (4',6-diamidino-2phenylindole) was supplied by Invitrogen (Carlsbad, CA, USA). Capmul $^{\circledR}$ GMO-50 (glyceryl monooleate) was a gift from Abitec (Commerce, CA, USA). Transcutol ${ }^{\circledR}$ HP (diethylene glycol monoethyl ether) was a gift from Gattefossé (Lyon, France).

\section{Solubility test}

The solubility of silymarin in the respective oils, surfactants, and cosurfactants used for preparing SNEDDS was determined. These included Capmul, Tween 20, Cremophor, and Transcutol. An excess amount of silymarin was added to $2 \mathrm{~mL}$ of the selected medium. The resulting mixture was shaken at $40^{\circ} \mathrm{C}$ for 24 hours, followed by centrifugation at $3,000 \times g$ for 10 minutes. The supernatant was filtered through a polyvinylidene fluoride membrane with a pore size of $0.45 \mu \mathrm{m}$ to remove any insoluble silymarin. The saturated solubility of silibinin in the silymarin extract was subsequently detected by high-performance liquid chromatography. The filtrate was diluted with the mobile phase before detection if necessary.

\section{Preparation of SNEDDS}

The optimized SNEDDS formulation for silymarin was modified from a previous study. ${ }^{13}$ The SNEDDS formulation used in this work consisted of Capmul GMO as the oil, Tween 20 and Cremophor RH40 (1:1, w/w) mixture as the surfactant, and Transcutol HP as a cosurfactant. The components of the formulation included $10 \%(\mathrm{w} / \mathrm{w})$ oil, $37.5 \%$ surfactant, $37.5 \%$ cosurfactant, and $15 \%$ silymarin. The oil and surfactant were respectively heated to $37^{\circ} \mathrm{C}$ before being added to the silymarin-Transcutol solution at room temperature. The mixture of oil and surfactants was stirred using a magnetic stirrer for 5 minutes. Next, $100 \mathrm{mg}$ of the mixture was transferred to a test tube, where it was diluted using double-distilled water or simulated gastric fluid (SGF) up to $10 \mathrm{~mL}$. SGF was prepared by adding $0.2 \mathrm{~g} \mathrm{NaCl}, 0.7 \mathrm{~mL} \mathrm{HCl}$, and $0.32 \mathrm{~g}$ pepsin $\mathrm{A}$ in water to a total volume of $100 \mathrm{~mL} .{ }^{14}$ The $\mathrm{pH}$ of this mixture was 1.2 . The mixture was gently stirred for 5 minutes to form the nanoemulsion dispersion. The nanoemulsion was examined for size, zeta potential, droplet number, and transmission electron microscopy.

\section{Size, zeta potential, and droplet number}

The mean droplet size and zeta potential of the prepared SNEDDS after dilution were evaluated by dynamic light scattering (Nano ZS90, Malvern Instruments, Malvern, UK). 
The resulting solution was gently shaken before light scattering analysis. The number of droplets formed after dilution was determined by NanoSight NS300 (Malvern).

\section{Transmission electron microscopy}

The size and morphology of SNEDDS after dilution were observed by transmission electron microscopy (H-7500, Hitachi, Tokyo, Japan). After gentle agitation, a drop of the nanoemulsion was deposited on a copper grid to form a thin-film specimen. The sample was stained using phosphotungstic acid $0.5 \%$. The sample was then examined and photographed by transmission electron microscopy.

\section{Animals}

Male Sprague-Dawley rats (350 450 g) were acquired from Lasco (Taipei, Taiwan). All animal procedures were performed in accordance with protocols approved prospectively by the Institute of Animal Care and Use Committee of Chang Gung Memorial Hospital, Chiayi, Taiwan. The rats were acclimatized for at least one week under a strict 12:12-hour light-dark cycle with ad libitum access to chow and water. The animals were fasted for 6-8 hours before surgery.

\section{Gastric bypass surgery}

An upper midline laparotomy was performed in each rat under anesthesia with 1\%-2\% isoflurane (Abbott Laboratories, Abbott Park, IL, USA) in oxygen. The animals underwent either an RYGB or a sham laparotomy. For RYGB animals, the body of the stomach was divided carefully to avoid injury of the peri-stomach vessels and nerves, leaving a 1-2 $\mathrm{mL}$ gastric pouch, including an area of glandular mucosa. The ends were sewn over with $6 / 0$ Prolene ${ }^{\circledR}$ running sutures (Ethicon, Somerville, NJ, USA). A 7 mm gastrostomy was performed, and a Roux-en-Y reconstruction was created with a $15 \mathrm{~cm}$ length Roux limb, and a biliopancreatic limb extending $10 \mathrm{~cm}$ from the ligament of Treitz. The proximal cut ends of the biliopancreatic limb were secured with $3 / 0$ Ethibond $^{\circledR}$ ties (Ethicon). They were then drained into the Roux limb using side-to-side anastomoses with a $1 \mathrm{~cm}$ lumen. All anastomoses were completed with 6/0 Prolene continuous sutures. The abdomen was lavaged before closure in two layers with 3/0 Ethibond. For the sham animals, after performing the laparotomy, the intestines were gently handled for 60 minutes before lavage and closure of the incision as above. Postoperatively, the animals were allowed to recover in a warm cage before returning to the postoperative room. All animals were given nalbuphine (Genovate, Hsinchu, Taiwan) at a dose of $0.1 \mathrm{mg} / \mathrm{kg}$ subcutaneously twice a day within
48 hours. The animals were maintained on a liquid diet for 2 days postoperatively, and supplied thereafter with distilled water and a chow diet ad libitum. Pharmacokinetic experiments were performed on day 7 after surgery.

\section{Pharmacokinetic study}

All rats were briefly anesthetized using 5\% isoflurane in oxygen. Isoflurane is an ideal anesthetic for intragastric study because it has a minimal effect on gastric motility. Silymarin $(600 \mathrm{mg} / \mathrm{kg})$ in aqueous suspension ( $1 \%$ carboxymethylcellulose sodium in water), PEG 400 solution, or SNEDDS, was administered orally to the rats. The oral formulations were administered via an oral intubation cannula, followed by administration of $1 \mathrm{~mL}$ of double-distilled water for washing the cannula. The percentage of silibinin in the silymarin was $24 \%$ (w/w) as determined by high-performance liquid chromatography. About $0.3 \mathrm{~mL}$ of the blood samples was collected through the tail vein into ethylenediaminetetraacetic acid-coated tubes at 0, 0.5, 1, 1.5, 2, 4, 8, 12 , and 24 hours. Blood samples were centrifuged at 3,000 $\times g$ and $4{ }^{\circ} \mathrm{C}$ for 10 minutes using a refrigerated centrifuging machine (Model 5500, Kubota, Tokyo, Japan). The plasma was withdrawn and stored at $-20^{\circ} \mathrm{C}$.

\section{Sample preparation and high- performance liquid chromatography apparatus}

The concentration of silibinin in plasma was measured after hydrolysis using a mixed enzyme solution containing sulfatase and $\beta$-glucuronidase. A $30 \mu \mathrm{L}$ aliquot of plasma was treated with $30 \mu \mathrm{L}$ of $\beta$-glucuronidase $(900 \mathrm{U} / \mathrm{mL})$ and sulfatase (300 U/mL) solution, buffered by sodium acetate $(\mathrm{pH} 5.0)$ at $37^{\circ} \mathrm{C}$ for one hour. The internal standard (narigenin $10 \mu \mathrm{g} / \mathrm{mL})$ in acetonitrile $(120 \mu \mathrm{L})$ was further added for protein sedimentation. Samples were centrifuged at 3,000 $\times g$ for 10 minutes (Model 3500, Kubota), and the supernatants were used for quantification. Chromatographic separation was performed using high-performance liquid chromatography (Agilent 1100 series, Agilent Technologies Inc, Santa Barbara, CA, USA). A $25 \mathrm{~cm}$ long, $4 \mathrm{~mm}$ inner diameter stainless revere phase C18 column (Macherey-Nagel, Duren, Germany) was used as the stationary phase. The mobile phase was an acetonitrile-pH 4.7 phosphoric acid (40:60) mixture at a flow rate of $1 \mathrm{~mL}$ per minute. The wavelength of the ultraviolet-visible detector was set at $288 \mathrm{~nm}$.

\section{Pharmacokinetic calculations}

Analysis of data from the pharmacokinetic study was done using WinNonlin Standard Edition version 1.1 (Pharsight, 
Mountain View, CA, USA). The peak silibinin concentration $\left(\mathrm{C}_{\max }\right)$ and time-to-peak concentration $\left(\mathrm{T}_{\max }\right)$ were obtained from the individual plasma concentration-time profiles. The area under the plasma concentration $\left(\mathrm{AUC}_{0 \rightarrow \infty}\right)$ was estimated using the trapezoidal rule, with extrapolation to infinity. The half-life, clearance, and mean residence time (MRT) were also calculated using the same software.

\section{Fluorescence and confocal microscopy}

The fluorescence-labeled formulations were prepared with Nile red as the dye at a concentration of $0.1 \%(\mathrm{w} / \mathrm{v})$. The formulations were administered orally to the rats. After 60 minutes, the animals were euthanized and the GI tract was harvested. After gentle irrigation with $0.9 \%$ sodium chloride, the specimens were put in embedding medium (OCT compound, Sakura, Tokyo, Japan) and rapidly frozen at $-4^{\circ} \mathrm{C}$. The frozen sections were cut serially at a thickness of 5-8 $\mu \mathrm{m}$. DAPI was used for nuclear staining. The slides were imaged using an Eclipse E600 microscope (Nikon, Tokyo, Japan), and the images were captured using a chargecoupled device camera (QICam Fast 1394, Qimaging, Surrey, BC, Canada) with QCapture Pro 7 software (Qimaging) in an auto exposure mode. Images of different color channels were merged after adjusting brightness and contrast in ImageJ (version 1.47). The slides were imaged by confocal microscopy (TSC SP5, Leica, Wetzlar, Germany), and the images were captured and processed with software Leica AF (version 2.6 .3 build 8173 ).

\section{Histological evaluation}

The acute toxicity of the PEG 400 solution and SNEDDS at the GI membrane was examined after a 24-hour treatment with the silymarin-containing formulations administered orally in normal rats. After the rats were euthanized, the mucosa of the stomach and jejunum was excised. The specimen was dehydrated using a 10\% buffered formaldehyde solution, and then embedded in paraffin wax. The samples were stained with hematoxylin and eosin or cyclooxygenase (COX)-2 antibody. The slides were imaged using a light microscope (BX51, Olympus, Tokyo, Japan), and microphotographs were captured using a digital camera (DP80, Olympus) with software (Cellsens version 1.7.1, Olympus) in auto exposure mode.

\section{Statistical analysis}

A statistical analysis of the differences between various treatments was done using the unpaired Student's $t$-test. A 0.01 or 0.05 level of probability was taken as the level of statistical significance. Analysis of variance was also used to compare the experimental data if necessary.

\section{Results \\ Physicochemical characterization of SNEDDS}

It is important to avoid precipitation after dilution of a drug in the GI tract. The materials used in SNEDDS should have a high solubilization capacity to confirm the dissolution of drugs. We selected Capmul GMO-50 (mainly glyceryl monooleate) as the oil phase, Tween 20 and Cremophor RH40 as surfactants, and Transcutol HP as the cosurfactant. The saturated solubility of silibinin in Capmul, Tween 20, Cremophor, and Transcutol was 333.41, 177.05, 99.44, and $469.33 \mathrm{mg} / \mathrm{mL}$, respectively. The combination of these additives in SNEDDS assured sufficient solubilization for silymarin loading. The silymarin-containing SNEDDS rapidly formed oil-in-water nanoemulsions upon dilution with aqueous medium in less than one minute. The appearance of the nanoemulsions was kept to be transparent after dilution. Table 1 shows the physicochemical properties of SNEDDS after aqueous dilution by 100 -fold. Following the dilution of SNEDDS with water, the mean droplet size of the nanoemulsions was $174 \mathrm{~nm}$, with a Gaussian distribution. The droplet size was also investigated in SGF to mimic the dispersion of SNEDDS in the gastric milieu. The average size of the nanoemulsions was slightly but significantly $(P<0.05)$ increased (to $190 \mathrm{~nm})$ in SGF. The polydispersity index was similar for SNEDDS in water and in SGF (about 0.4). SNEDDS in water showed a negative charge of $-19 \mathrm{mV}$. Reconstitution of SNEDDS in SGF reached a zeta potential near zero. The number of droplets in water was greater than that in SGF.

Figure 1 shows the distribution and morphology of the nanoemulsion droplets as observed by transmission electron microscopy. The droplets appeared to be of a spherical shape without aggregation (Figure 1A). The size of the droplets was uniform. Figure $1 \mathrm{~B}$ shows the images at higher magnification

Table I Characterization of self-nanoemulsifying drug delivery systems in double-distilled water and simulated gastric fluid by mean diameter, zeta potential, and particle numbers

\begin{tabular}{lll}
\hline Characteristics & $\begin{array}{l}\text { Double-distilled } \\
\text { water }\end{array}$ & $\begin{array}{l}\text { Simulated gastric } \\
\text { fluid }\end{array}$ \\
\hline Size $(\mathrm{nm})$ & $173.5 \pm 1.0$ & $190.0 \pm 2.5$ \\
Polydispersity index & $0.39 \pm 0.004$ & $0.42 \pm 0.04$ \\
Zeta potential $(\mathrm{mV})$ & $-18.9 \pm 0.3$ & $-0.6 \pm 0.3$ \\
Droplet number $($ droplets $/ \mathrm{mL})$ & $1.3 \times 10^{11}$ & $1.0 \times 10^{11}$ \\
\hline
\end{tabular}

Note: Each value represents the mean \pm standard deviation $(n=3)$. 

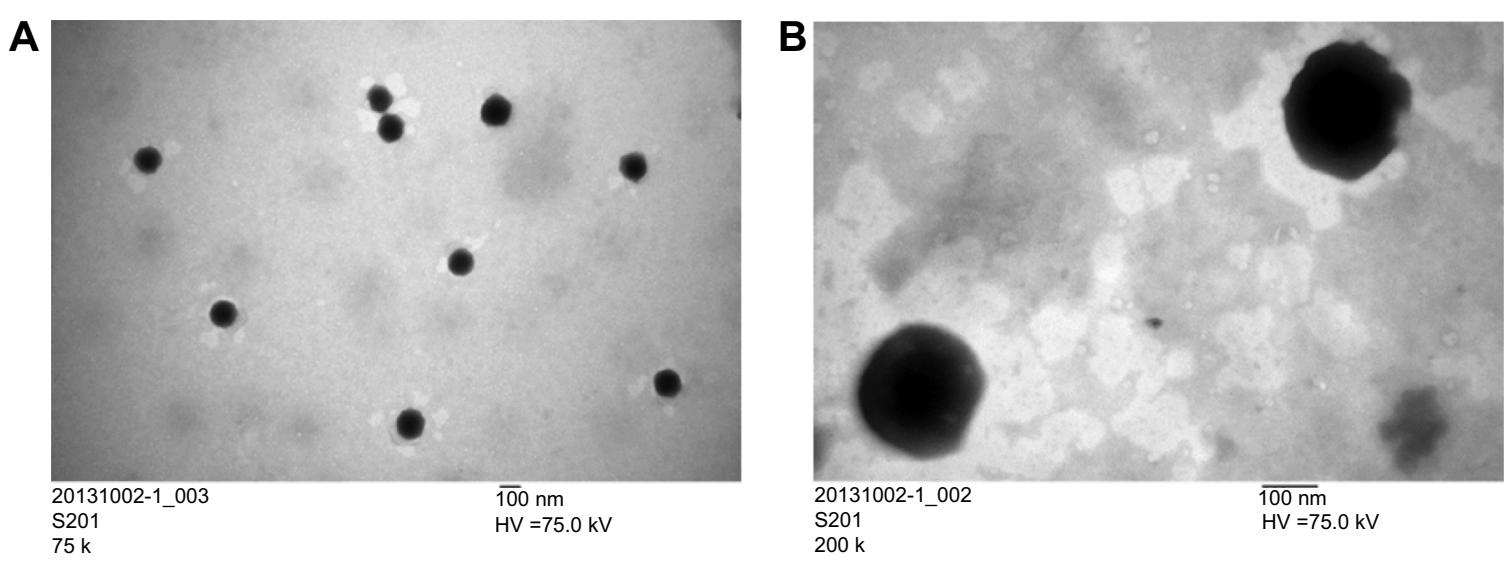

Figure I Transmission electron micrographs of self-nanoemulsifying drug delivery systems at magnifications of $(\mathbf{A}) 75,000 \times$ and $(\mathbf{B}) 200,000 \times$. The scale bar is I00 nm.

(200,000×). A diameter of about $200 \mathrm{~nm}$ was observed, which was consistent with the size determined by dynamic light scattering.

\section{Gastric bypass surgery}

We performed an RYGB procedure on rats weighing $350-450 \mathrm{~g}$. Figure $2 \mathrm{~A}$ shows an intraoperative image of the RYGB rat. This surgical procedure created a gastric pouch and inserted a short Roux alimentary limb from the distal small bowel, which was anastomosed to the stomach and the proximal small bowel. The postoperative GI tract was exposed outside the abdominal cavity for a clearer view, as shown in Figure 2B. The gastric pouch was connected to the Roux limb (arrow in Figure 2B). The small intestine was reconnected to bypass the duodenum. Finally, the biliopancreatic limb and Roux limb were joined together for drainage of the biliary-pancreatic digestive juices (triangle in Figure 2B). The body weight of the RYGB rats was recorded postoperatively, as demonstrated in Figure 2C. The results were compared with the body weight of the rats in the sham surgery group. Rats in the sham group showed a decrease in body weight during the first 2 days after surgery. Their weight then steadily and continuously increased until 11 days postoperatively. Conversely, the body weight of the RYGB animals progressively decreased after surgery. The RYGB rats lost an average of $25 \%$ body weight by day 11 after surgery.

\section{Pharmacokinetic study}

Oral administration of SNEDDS was investigated in normal and RYGB rats and compared with the absorption of silymarin suspension and PEG 400 solution. Plasma silibinin levels were detected. Silibinin is the primary component in silymarin. Figure $3 \mathrm{~A}$ shows the difference in average plasma concentration of silibinin after oral application of three silymarin formulations at a dose of $600 \mathrm{mg} / \mathrm{kg}$ in normal rats. Similar curves were observed for PEG 400 solution and SNEDDS, which showed a higher silibinin concentration compared with the suspension. The pharmacokinetic parameters are summarized in Table 2. The $\mathrm{C}_{\text {max }}$ and AUC of SNEDDS were $11.84 \mu \mathrm{g} / \mathrm{mL}$ and $163.42 \mu \mathrm{g} \cdot h$ hour $/ \mathrm{mL}$, respectively. SNEDDS showed no difference $(P>0.05)$ from the silymarin solution for values of $\mathrm{C}_{\max }$ and $\mathrm{AUC}$, which were 1.6-fold higher than the values obtained with the aqueous suspension. The half-life of the suspension (15.03 hours) was relatively longer $(P<0.05)$ than that for PEG 400 (10.45 hours) and SNEDDS (9.04 hours). A consequence of this was a higher MRT $(P<0.05)$ for the suspension as compared with the other formulations.

Figure 3B shows the plasma concentration-time profiles for the silymarin vehicles in RYGB rats. Pharmacokinetic parameters obtained by WinNonlin are listed in Table 3 . The peak plasma silibinin level occurred at $2-3$ hours post-dosing for PEG 400 and SNEDDS. A secondary peak was detected at about 10 hours after gavage with SNEDDS. The suspension showed a delayed $\mathrm{T}_{\text {max }}$ of 4.71 hours. A secondary peak of 12 hours was also observed for the suspension. The $\mathrm{C}_{\max }$ of silibinin for PEG 400 and SNEDDS was 13.77 and 13.65 $\mu \mathrm{g} / \mathrm{mL}$, respectively, whereas for the aqueous suspension it was only $5.37 \mu \mathrm{g} / \mathrm{mL}$. The AUC of silibinin in RYBG rats receiving SNEDDS $(180.15 \mu \mathrm{g} \cdot$ hour $/ \mathrm{mL})$ was higher in comparison with PEG $400(123.20 \mu \mathrm{g} \cdot \mathrm{hour} / \mathrm{mL})$ and the suspension $(72.77 \mu \mathrm{g} \cdot$ hour $/ \mathrm{mL})$. This difference was statistically significant, as seen in Figure 3C. The AUC increased by 2.5 and 1.5 times following SNEDDS administration when compared with the silymarin suspension and solution, respectively. There was no significant difference in half-life or MRT between the three formulations in RYGB rats. Clearance 

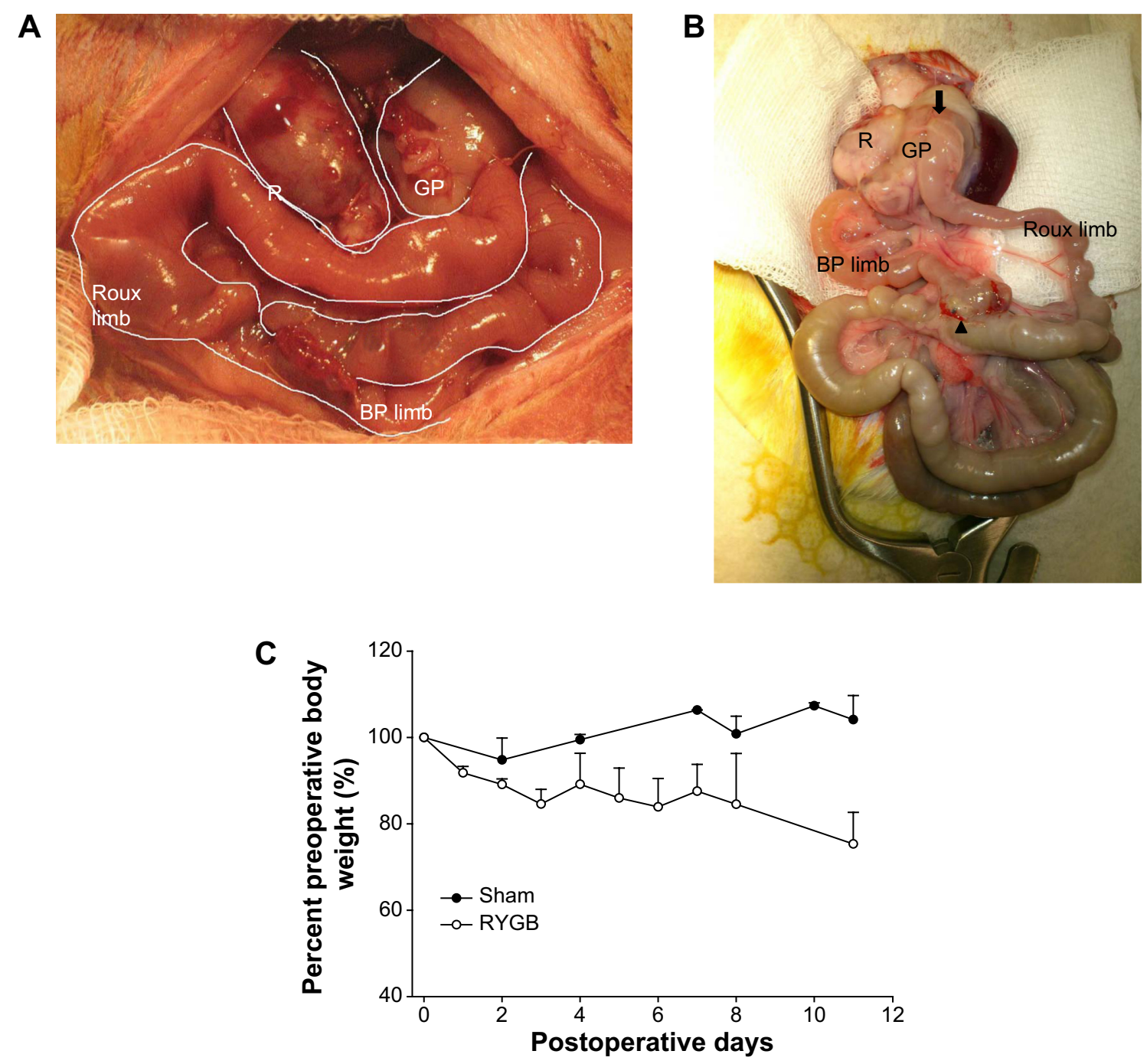

Figure 2 RYGB in the rat model.

Notes: (A) Intraoperative imaging of RYGB rat. (B) View of gastrointestinal tract exposed outside the abdominal cavity. The arrow indicates the connection of the gastric pouch to the Roux limb. The triangle indicates the connection of the biliopancreatic limb and the Roux limb. (C) Change in body weight of sham rats and RYGB rats after surgery. Abbreviations: R, remaining stomach; GP, gastric pouch; BP limb, biliopancreatic limb; RYGB, Roux-en-Y gastric bypass.

of the suspension $(1,978.91 \mathrm{~mL} / \mathrm{hour} / \mathrm{kg})$ was significantly higher than that of PEG $400(1,168.78 \mathrm{~mL} /$ hour $/ \mathrm{kg})$ and the nanocarriers $(986.06 \mathrm{~mL} / \mathrm{hour} / \mathrm{kg})$, and there was no difference $(P>0.05)$ between the data of PEG 400 and nanoemulsions. The data for the RYGB animals demonstrated that SNEDDS resulted in enhanced absorption of silibinin compared with the suspension and solution.

\section{Fluorescence microscopy}

In order to explore the permeability of silymarin via the gastric and intestinal mucosa, the distribution of PEG 400 solution and SNEDDS containing Nile red as the dye was monitored at the GI membrane by fluorescence microscopy. Figure 4A shows the macroscopic observations of the GI tract in RYGB rats receiving PEG 400 or SNEDDS.
The arrows in Figure 4A are the sites of gastrojejunostomy. The pouch and alimentary limb in the SNEDDS-treated rat were significantly redder in color than those in the PEG 400-treated rat. The red color could have originated from the Nile red. Vessels in the GI mucosa were stained red after delivery of SNEDDS. This observation initially approved the better absorption of SNEDDS compared with the solution.

Figure 4B shows the stomach of normal rats on fluorescence microscopy following oral delivery of Nile red-loaded PEG 400 and SNEDDS. The triangles in the imaging indicate the lumen. The red fluorescence in the PEG 400-treated group was mainly derived from the rugae of the stomach. Nile red in PEG 400 penetrated into the mucosa. On the other hand, Nile red in SNEDDS not only adhered to the superficial epithelium 
A

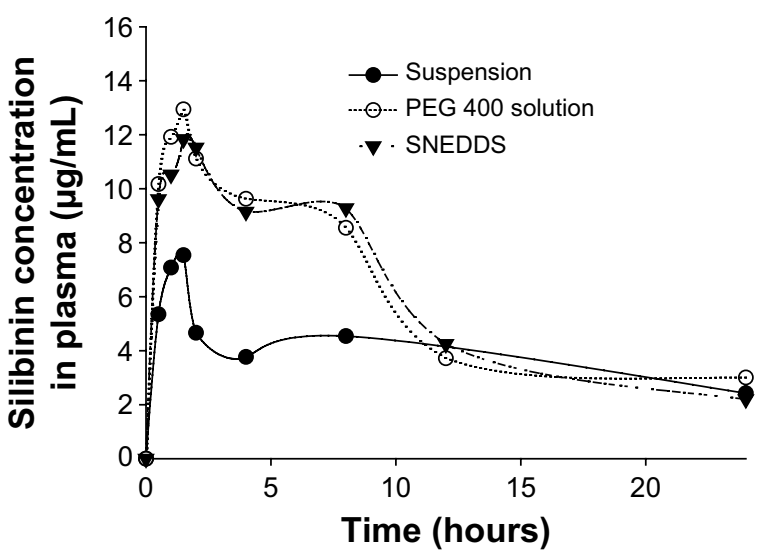

B

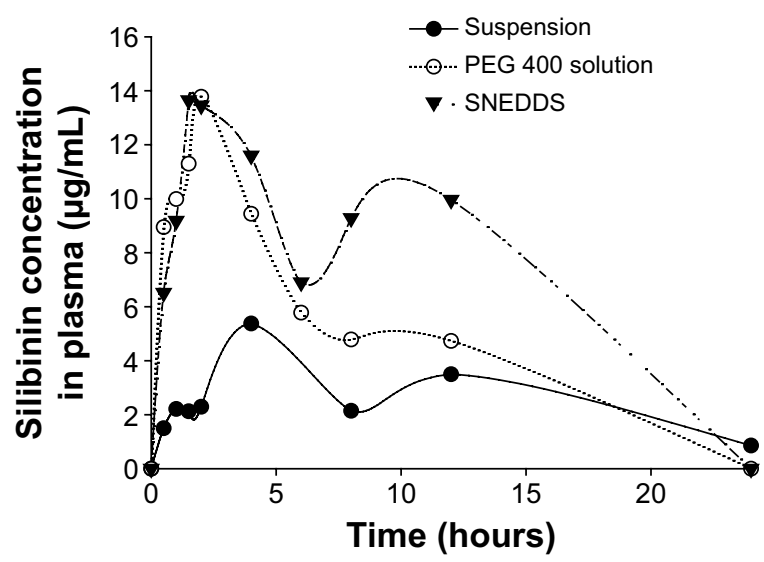

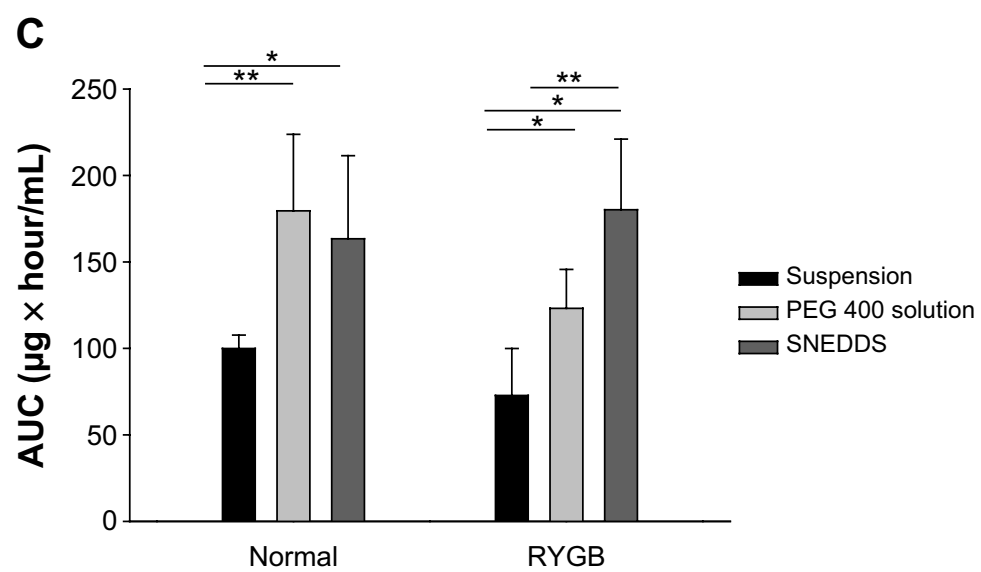

Figure 3 Pharmacokinetic profiles of silibinin after oral administration of aqueous suspension, PEG 400 solution, and SNEDDS.

Notes: (A) Mean plasma concentration of silibinin versus time curves in normal rats. (B) Mean plasma concentration of silibinin versus time curves in RYGB rats. (C) Comparison of area under curve (AUC) of the plasma concentration-time curves. $* P<0.05$, $* * P<0.01$.

Abbreviations: RYGB, Roux-en-Y gastric bypass; PEG, polyethylene glycol; SNEDDS, self-nanoemulsifying drug delivery systems.

but also was transported deeply into the lamina propria. Figure $4 \mathrm{C}$ shows the fluorescence imaging of the intestine after treatment with PEG 400 and SNEDDS in normal rats. As with the stomach, almost no Nile red penetrated into the intestinal mucosa. Most of the fluorescence stayed outside

Table 2 Pharmacokinetic parameters of silibinin $(144 \mathrm{mg} / \mathrm{kg})$ after oral administration of silymarin from suspension, PEG 400 solution, and SNEDDS in control rats

\begin{tabular}{llll}
\hline Parameter & Suspension & PEG 400 & SNEDDS \\
\hline $\mathrm{C}_{\text {max }}(\mu \mathrm{g} / \mathrm{mL})$ & $7.54 \pm 0.79$ & $12.94 \pm 6.57$ & $\mathrm{II} .84 \pm 6.2 \mathrm{I}$ \\
$\mathrm{T}_{\text {max }}$ (hours) & $1.33 \pm 0.29$ & $1.67 \pm \mathrm{I} .2 \mathrm{I}$ & $\mathrm{I} .3 \mathrm{I} \pm 0.65$ \\
$\mathrm{AUC}$ ( $\mu \mathrm{g} \cdot$ hour $/ \mathrm{mL})$ & $99.97 \pm 7.73$ & $179.55 \pm 44.2 \mathrm{I}$ & $163.42 \pm 48.05$ \\
Half-life (hours) & $15.03 \pm \mathrm{I} .42$ & $10.45 \pm 2.25$ & $9.04 \pm 3.37$ \\
Clearance $(\mathrm{mL} /$ hour $/ \mathrm{kg})$ & $823.01 \pm 86.64$ & $801.99 \pm 230.03$ & $88 \mathrm{I} .15 \pm 222.49$ \\
MRT (hours) & $24.16 \pm 2.42$ & $15.66 \pm 5.46$ & $12.68 \pm 5.17$ \\
\hline
\end{tabular}

Note: Each value represents the mean \pm standard deviation $(n=5-10)$. Abbreviations: $\mathrm{C}_{\max }$, maximum plasma concentration; $\mathrm{T}_{\max }$, time to reach $\mathrm{C}_{\max }$; AUC, area under the plasma concentration-time curve; MRT, mean residence time; PEG, polyethylene glycol; SNEDDS, self-nanoemulsifying drug delivery systems. the villi. SNEDDS could deliver Nile red into the upper two thirds of the villi. Some fluorescence was also observed in the lumen for the SNEDDS group. With respect to the RYGB rats, microphotographs of the stomach after application of PEG 400 or SNEDDS are shown in Figure 4D. Nile red from PEG 400 had reached the superficial mucosa, including the lamina propria. It can be seen that the distribution of red fluorescence was much deeper from SNEDDS than from the solution. Nile red was distributed throughout the full thickness of the mucosa, including the lamina propria and the glandular region. PEG 400 delivered Nile red to the upper one third height of the villi in the RYGB rats, as shown in Figure 4E. Strong fluorescence was observed at the mucosal surface of the intestine after treatment with SNEDDS. This indicates the ability of SNEDDS to adhere to the intestine membrane, prolonging the residence time. Nile red in SNEDDS diffused into the upper two thirds of the villi in the RYGB animals. This result was consistent with that of SNEDDS delivery into the villi of normal rats. 
Table 3 Pharmacokinetic parameters of silibinin ( $444 \mathrm{mg} / \mathrm{kg}$ ) after oral administration of silymarin from suspension, PEG 400 solution, and SNEDDS in RYGB rats

\begin{tabular}{llll}
\hline Parameter & Suspension & PEG 400 & SNEDDS \\
\hline $\mathrm{C}_{\text {max }}(\mu \mathrm{g} / \mathrm{mL})$ & $5.37 \pm 4.72$ & $13.77 \pm 6.72$ & $13.65 \pm 5.59$ \\
$\mathrm{~T}_{\text {max }}$ (hours) & $4.71 \pm 2.50$ & $2.0 \pm 0$ & $2.83 \pm 1.29$ \\
$\mathrm{AUC}(\mu \mathrm{g}$.hour $/ \mathrm{mL})$ & $72.77 \pm 27.18$ & $123.20 \pm 22.44$ & $180.15 \pm 40.86$ \\
Half-life (hours) & $8.46 \pm 3.86$ & $6.39 \pm 1.75$ & $8.45 \pm 2.22$ \\
Clearance $(\mathrm{mL} /$ hour $/ \mathrm{kg})$ & $1,978.91 \pm 606.38$ & $1,168.78 \pm 394.34$ & $986.06 \pm 255.57$ \\
MRT (hours) & $12.46 \pm 4.48$ & $9.91 \pm 3.71$ & $11.26 \pm 3.90$ \\
\hline
\end{tabular}

Note: Each value represents the mean \pm standard deviation $(n=5-10)$.

Abbreviations: $C_{\text {max }}$, maximum plasma concentration; $T_{\text {max }}$, time to reach $C_{\text {max }}$; $A U C$, area under the plasma concentration-time curve; MRT, mean residence time; $R Y G B$, Roux-en-Y gastric bypass; PEG, polyethylene glycol; SNEDDS, self-nanoemulsifying drug delivery systems.

A
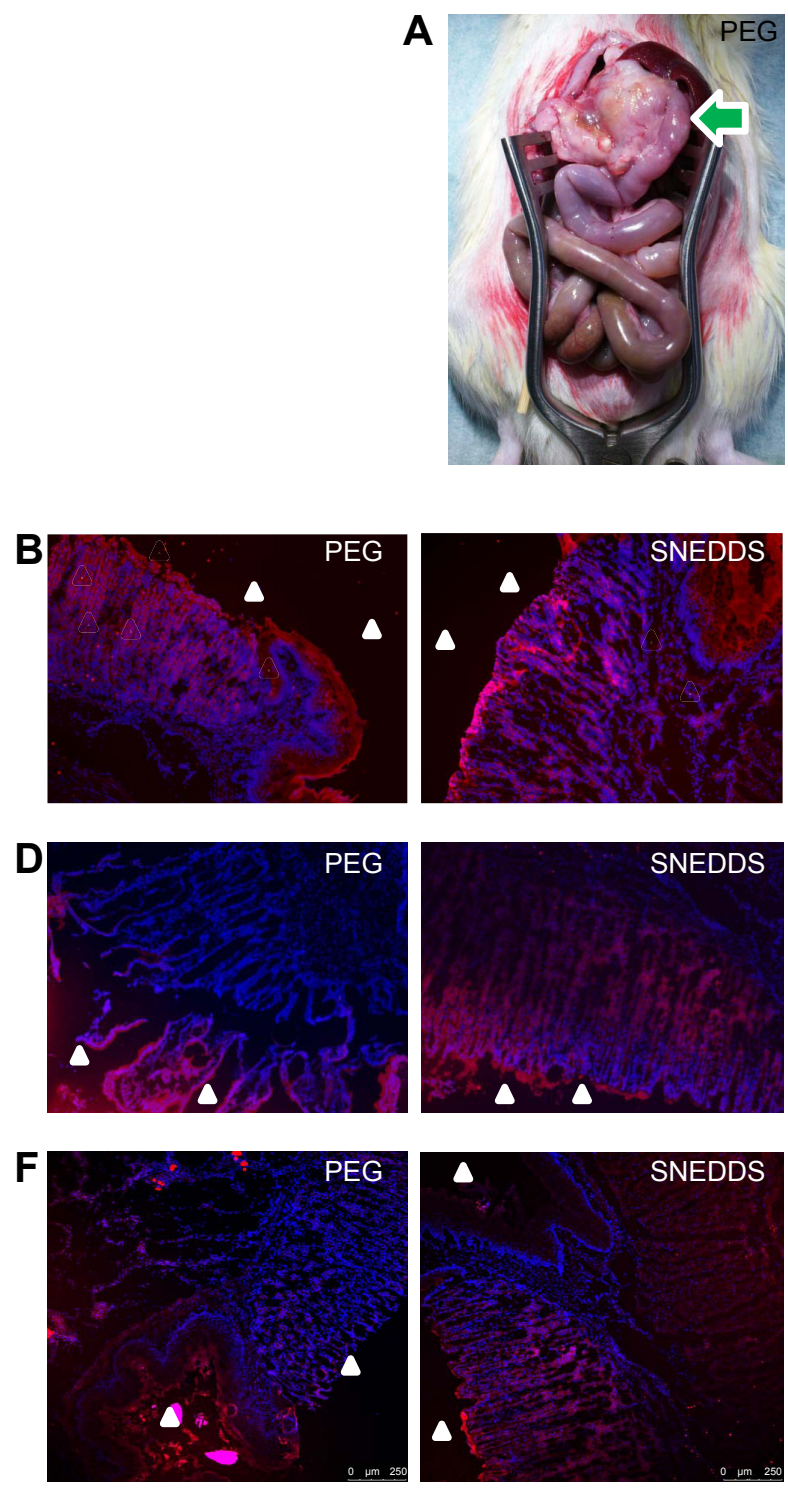
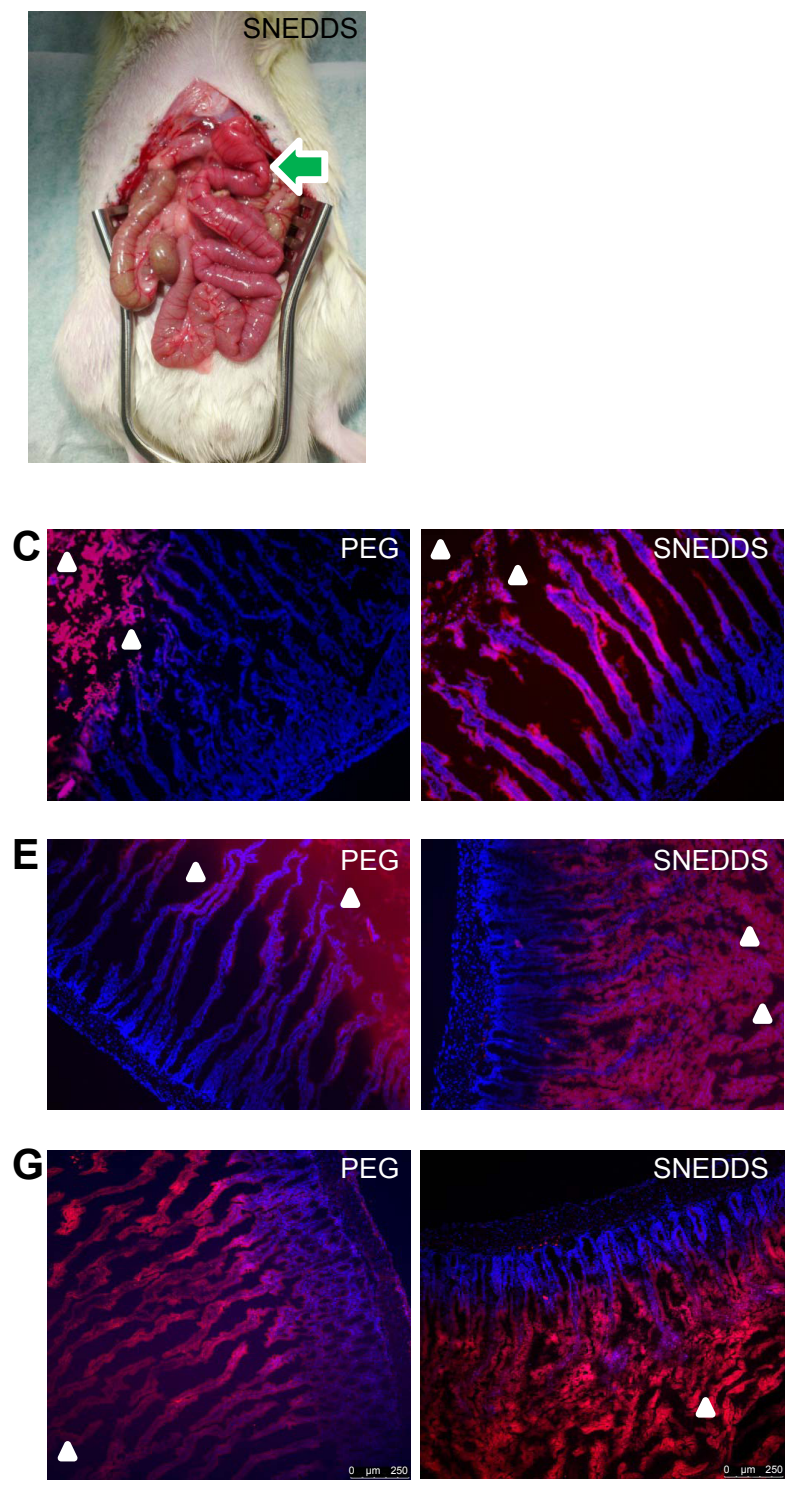

Figure 4 Fluorescence imaging of stomach and intestine of rats after oral administration of Nile red-containing PEG 400 solution and SNEDDS.

Notes: (A) Macroscopic observation of GI tract exposed outside the abdominal cavity after oral administration of PEG 400 and SNEDDS in RYGB rats. The arrows indicate the connection of gastric pouch to the Roux limb. (B) Microscopic observation of stomach (I00x) after oral administration of PEG 400 and SNEDDS in normal rats. (C) Microscopic observation of intestine (100X) after oral administration of PEG 400 and SNEDDS in normal rats. (D) Microscopic observation of stomach (I00X) after oral administration of PEG 400 and SNEDDS in RYGB rats. (E) Microscopic observation of intestine (I00x) after oral administration of PEG 400 and SNEDDS in RYGB rats. (F) Confocal imaging of stomach (100X) after oral administration of PEG 400 and SNEDDS in RYGB rats. (G) Confocal imaging of intestine (I00X) after oral administration of PEG 400 and SNEDDS in RYGB rats. Triangles in (B-G) indicate the lumens.

Abbreviations: RYGB, Roux-en-Y gastric bypass; PEG, polyethylene glycol; SNEDDS, self-nanoemulsifying drug delivery systems. 
In order to confirm the results of fluorescence microscopy, we used confocal laser scanning microscopy to examine the distribution of Nile red in the stomach and intestine of the RYGB rat. Figure 4F shows confocal images of the stomach on treatment with the solution and nanosystems. As in Figure 4B, the red signal is mainly located in the lumen after delivery of PEG 400. Some signals could be detected inside the mucosa. The fluorescence intensity of SNEDDS in the columnar epithelium seemed stronger than that of PEG 400. Deeper distribution in the mucosa was also observed in the SNEDDS sample. As Figure $4 \mathrm{G}$ shows, the PEG 400 vehicle delivered a significant amount of Nile red into the villi. A similar phenomenon was observed in the intestines treated with the nanocarriers. Nile red in SNEDDS largely accumulated in the lumen near the mucosal surface, which is consistent with the observations made on fluorescence microscopy.

\section{Histological evaluation}

The safety and tolerance of the oral nanocarriers is an important issue to consider. Figure $5 \mathrm{~A}$ and $\mathrm{B}$ show representative examples of hematoxylin and eosin-stained images of the stomach and intestine, respectively. The triangles and arrows in Figure 5A indicate squamous epithelium and columnar epithelium, respectively, in the esophagus. The gastric mucosa appears normal after treatment with PEG 400, except for the presence of some inflammatory cells at the gastroesophageal junction. A similar phenomenon was observed for the SNEDDS group. The inflammation at the gastroesophageal junction induced by SNEDDS was milder than in that seen after treatment with the PEG 400 solution. The triangles in Figure 5B represent the intestinal lumen. The intestinal membrane had an almost normal appearance after treatment with the silymarin-containing PEG 400 solution. Mild to moderate chronic inflammation was observed in the lamina propria, which could be lessened by administration of SNEDDS. Expression of COX-2 in the stomach and intestine after administration of silymarin is shown in Figure 5C and D, respectively. COX-2 expression in the gastric mucosa with PEG 400 was greater than that with SNEDDS (Figure 5C). In contrast, higher COX-2 expression in the intestinal membrane was seen with SNEDDS than with PEG 400 solution.

\section{Discussion}

Poor dissolution and bioavailability in patients receiving silymarin therapy remain clinical issues. This low oral efficiency is worsened in patients undergoing gastric bypass surgery.
Silymarin preparations with improved bioavailability are needed. The aim of this work was to develop novel SNEDDS that can improve the delivery of oral silymarin and prolong its efficacy. The experimental results showed that silymarin loading in SNEDDS provided benefits in terms of increased GI permeability and oral absorption. The irritating effect of SNEDDS at the GI membrane could be tolerated. Most studies involving self-emulsifying nanosystems have used normal animals to examine bioavailability. Nevertheless, the patients with some abnormalities are the actual targets for SNEDDS administration. We used RYGB rats to evaluate the effect of SNEDDS for simulating the actual clinical condition.

Silibinin is a compound with limited lipophilic characteristics; even its solubility in water is very low. ${ }^{15}$ Silibinin showed high solubility in PEG 400 (304.92 mg/mL), probably because of the ability of silibinin to form hydrogen bonds with polyethylene oxide moieties. Similarly, Cremophor composed of polyethylene oxide provided high solubilization to silibinin. All additives in SNEDDS contain hydroxyl groups, contributing to sufficient solubility for silibinin. The droplet size of the nanosystems was $<200 \mathrm{~nm}$, indicating good self-emulsifying efficiency in an aqueous medium. Emulsification with multiple surfactants rather than a single surfactant produces systems with smaller and more homogeneous globules. ${ }^{16}$ The nanoemulsions could be defined as SNEDDS according to the measured size after spontaneous dilution. Silymarin-loaded SNEDDS were negatively charged after dilution in water. This was possibly due to the presence of monoglycerides and diglycerides in Capmul. Free fatty acids derived from hydrolysis of glycerides can render the anionic fractions in nanocarriers. ${ }^{17}$ Various ions are present in the physiological environment of the GI tract. These ions minimize the surface charge of nanoemulsions generated from self-emulsifying systems. ${ }^{18}$ This is the reason that SGF shielded the negative charge of SNEDDS. The zeta potential of near 0 results in insufficient repulsion between droplets and subsequent aggregation, confirming the larger droplets in SGF as compared with water. A previous study ${ }^{19}$ also confirmed that the stomach favors nanoparticulate fusion because of its acidity and high ionic strength.

RYGB surgery caused a weight loss of $25 \%$ in the experimental rats by day 11 postoperatively. For rats, 10 13 days approximates one human year. ${ }^{20}$ Weight loss in obese patients undergoing gastric bypass surgery was reported to be $32 \%$ of initial body weight after $1-2$ years. ${ }^{21}$ This rate of reduction was similar to that in the rats used in the present work. Reduced food intake and malabsorption led to weight loss. Higher energy expenditure after RYGB than after the sham 

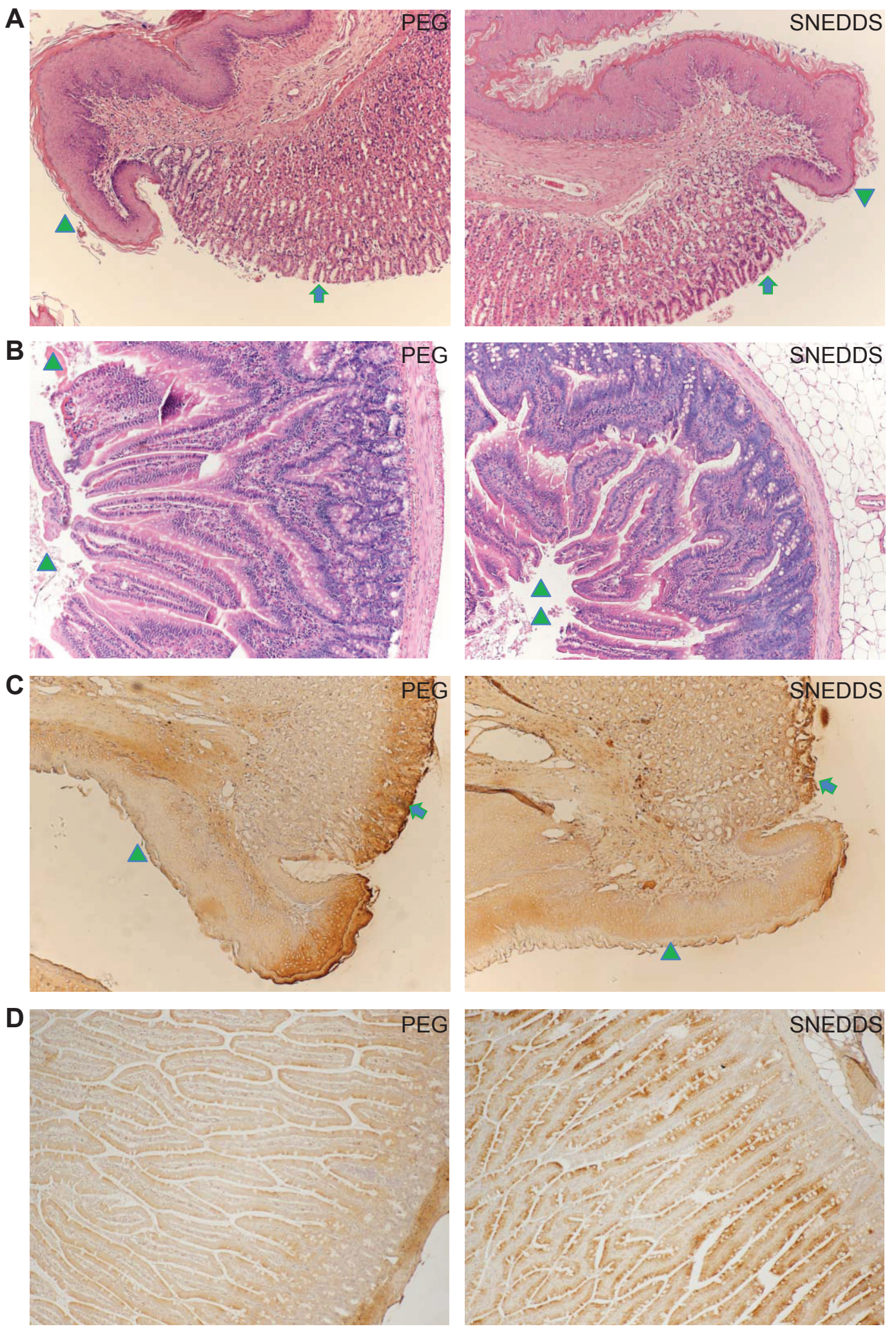

Figure 5 Histopathological observation of stomach and intestine of rats after oral administration of Nile red-containing PEG 400 solution and SNEDDS.

Notes: (A) H\&E staining of stomach (40X) after oral administration of PEG 400 and SNEDDS in normal rats. (B) H\&E staining of intestine (I00X) after oral administration of PEG 400 and SNEDDS in normal rats. The triangles indicate the lumens. (C) COX-2 staining of stomach (40X) after oral administration of PEG 400 and SNEDDS in normal rats. (D) COX-2 staining of intestine (100X) after oral administration of PEG 400 and SNEDDS in normal rats. The triangles and arrows in (A-C) indicate esophagus squamous epithelium and columnar epithelium, respectively.

Abbreviations: COX-2, cyclooxygenase-2; H\&E, hematoxylin and eosin; PEG, polyethylene glycol; SNEDDS, self-nanoemulsifying drug delivery systems. 
operation was another mechanism of weight loss. ${ }^{22}$ The sham operation caused weight loss in the first 2 days, which may have been due to the effect of anesthesia and postoperative stress. ${ }^{23}$

Silymarin in aqueous suspension, PEG 400 solution, and SNEDDS were orally administered to RYGB rats in order to examine plasma concentrations. The normal rat but not the sham rat was employed in this experiment for comparison. Previous studies ${ }^{24,25}$ indicate that the sham surgical procedure generally does not influence pharmacokinetic parameters. It can be observed that the half-life and MRT of silibinin from the suspension and the solution were significantly reduced in RYGB rats when compared with normal rats. Clearance was increased by RYGB. Bypass surgery also decreased the AUC for the suspension and solution, although no significant difference was found. This decrease in bioavailability and other pharmacokinetic parameters was not observed for SNEDDS. Because of the lengthened gastric and intestinal bypass, the diminished drug or nutrient absorption is anticipated. ${ }^{26}$ The $\mathrm{pH}$ of the stomach would be elevated by RYGB due to deficient secretion of gastric juices. ${ }^{27}$ Silibinin is rapidly absorbed in the stomach after oral ingestion. ${ }^{28}$ This molecule exists in a nonionic form in an acidic environment. The percentage existing in the ionic form increases with increasing $\mathrm{pH}$. Since nonionic molecules are better able to penetrate the GI membrane, the more-alkaline stomach by RYGB might lead to diminished absorption of silibinin. This phenomenon was not significant for SNEDDS since the nanodroplets could form a protective shell to avoid direct contact with gastric fluid. GI motility is reported to be increased by RYGB, ${ }^{29}$ so there may be adequate time for complete drug dissolution and absorption. The drug residence time in the body is thus shortened. The MRT profiles confirm such inference.

The high AUC value in the case of SNEDDS confirms that that the nanosystems used in this work can improve the bioavailability of silibinin. The extent and rate of absorption of drugs with poor solubility in water is highly dependent upon the formulations selected. The pharmacokinetic study of silymarin in powder form indicated a very low silibinin plasma concentration. ${ }^{13}$ The drug should be in a dissolved form before GI absorption. SNEDDS rapidly emulsified in water after dilution. The drug shows a high dissolution rate when solubilized in SNEDDS. ${ }^{30}$ The nanodroplets provide a large total surface area for rapid drug release and absorption, resulting in improved bioavailability. Since RYGB limits the space available for absorption in the stomach, rapid dissolution and absorption by SNEDDS to ameliorate bioavailability was especially important in the RYGB group. This is why SNEDDS were associated with a more favorable AUC in
RYGB rats than in normal rats. Silymarin was fully solubilized in PEG 400. The complete solubilization by PEG 400 and SNEDDS suggested that the mechanism for the improvement in bioavailability brought about by the nanocarriers involved not only the dissolution rate. PEG 400 has strongly hydrophilic characteristics, ${ }^{18}$ leading to difficulty with regard to partitioning into the lipophilic GI membrane. When dissolved in oil droplets, the drug can be absorbed directly by the membrane via fusion or interaction. ${ }^{31}$ Enhanced permeability is another possibility for the improved bioavailability seen with SNEDDS. Some surfactants can penetrate into the GI mucosa and disrupt the lipid bilayer, thereby increasing the rate of oral absorption. Cremophor and Transcutol used in our SNEDDS have been reported to have the ability to enhance membrane permeability. ${ }^{32}$ Monoglycerides and oleic oil are also known to increase membrane permeability. ${ }^{33,34}$ The structure of Capmul relates to monoglycerides and oleic oil. The results of fluorescence microscopy appear to correlate with the pharmacokinetic profiles, indicating a deeper and broader distribution of SNEDDS than the PEG 400 vehicle.

There was a significant second peak of plasma silibinin concentration in the RYGB rats receiving SNEDDS. In fact, most of the silymarin formulations tested had a high silibinin concentration at a late stage (8-12 hours) after administration. This could be attributable to the enterohepatic circulation, an important recycling pathway for silymarin. ${ }^{35}$ Two different absorption sites are another occasion of double peaks. Silymarin is rapidly absorbed in the stomach after administration, and well absorbed in the middle segment of the intestine. ${ }^{28,36}$ As compared with PEG 400, the SNEDDS increased the bioavailability of silibinin, mainly due to the increase of AUC at the late stage of administration. Both silymarin and silibinin undergo extensive first-pass metabolism in the liver. ${ }^{37}$ The nanodroplets containing oils and emulsifiers can be incorporated with lipoproteins and chylomicrons in the intestine. Such mixtures are largely taken up via the lymphatics, thus minimizing the first-pass effect. ${ }^{38}$ Previous studies ${ }^{8,39}$ have demonstrated enhanced lymphatic bioavailability by longchain fatty acids and glycerides. Capmul may fit this criterion. Cremophor RH40 in SNEDDS is also reported to prolong the duration of drug absorption, especially in the lower portion of the GI tract. ${ }^{40} \mathrm{~A}$ well-defined protocol is needed to elucidate the effect of lymphatic absorption for SNEDDS further.

The nanodroplets in SNEDDS have bioadhesive properties due to the increased viscosity. ${ }^{41}$ The viscous oil and surfactant system relative to water may contribute to adherence of SNEDDS to the mucosa due to the stickiness of the nanodispersions. This characteristic allows intimate contact with the 
membrane, prolonging the residence time in the GI tract and enhancing absorption. ${ }^{42}$ Fluorescence imaging demonstrated that the SNEDDS could adhere to the intestinal epithelium to a great extent. Bile salts in the GI tract facilitate degradation of lipids by enzymes such as lipase. ${ }^{43}$ This lipolysis digests the composition of lipid-based nanosystems, leading to loss of efficient delivery by the nanoparticles. The RYGB procedure involves construction of a biliopancreatic limb that diverts bile and pancreatic fluids from the proximal to the distal gut. This bypass significantly delays the interaction between lipolytic enzymes and ingested materials. ${ }^{44}$ This effect might delay the breakdown of SNEDDS in the intestine. Thus, the efficient dissolution and absorption by SNEDDS could be maintained for a longer time. The superior absorption of SNEDDS over PEG 400 was observed for RYGB rats but not for normal rats. This demonstrates the importance of lipolysis in delivery of a nanoemulsion. Efflux of P-glycoprotein and cytochrome P450 metabolism play a critical role in limiting GI absorption. Some lipids and surfactants in self-emulsifying systems, such as monoglycerides, Cremophor, and polysorbates, are known to inhibit P-glycoprotein and cytochrome P450. ${ }^{9,45}$ Most of the transporters and efflux pumps are present in the proximal small intestine, which is bypassed by RYGB. ${ }^{30}$ Inhibition of P-glycoprotein and cytochrome P450 by SNEDDS for enhanced availability may be ruled out as the predominant mechanism.

Another observation of the pharmacokinetic parameters in RYGB animals is a higher intersubject variability of aqueous suspension compared with silymarin solution and SNEDDS. Drugs with poor water solubility in undissolved form always undergo erratic absorption and show considerable variation in bioavailability. ${ }^{46}$ The surgery also produced intersubject variability in GI physiology. The drugs in their dissolved form spread uniformly in the GI tract. More reproducible absorption could be achieved with less dependence on different physiological states. SNEDDS can distribute and release their encapsulated drug in a controlled manner for decreasing the intersubject variability. ${ }^{47}$

Large amounts of surfactant and cosurfactant in selfemulsifying nanosystems can be irritating and toxic to the GI mucosa. We selected nonionic surfactants to produce SNEDDS since they are considered less toxic than ionic surfactants and are less affected by changes in $\mathrm{pH}$ and ionic strength. ${ }^{48}$ The histological examination showed greater inflammation of the GI membrane in response to PEG 400 than to SNEDDS, although the degree of inflammatory was low. GI symptoms are the most common adverse effects of administration of silymarin, occurring with a frequency of $2 \%-10 \% .{ }^{49}$ Inclusion of silymarin in oil droplets in SNEDDS might decrease the extent of direct contact between the drug with the mucosa, resulting in less inflammation. However, it should be noted that SNEDDS induced more COX-2 expression in the intestine than did PEG 400. Levels of COX-2 increase dramatically during inflammation. Upregulation of COX-2 plays an important role in pathological behavior characterized by increased prostaglandin production. ${ }^{50}$ This suggests that SNEDDS might increase prostaglandin levels in intestinal tissue without eliciting inflammatory infiltration as observed on hematoxylin and eosin staining. Ingredients in SNEDDS, such as Cremophor RH40, might be toxic to biomembranes. Kiss et $\mathrm{al}^{51}$ demonstrate that Cremophor RH40 is toxic to epithelial cells, although this effect is minor compared with the cytotoxicity of Cremophor EL. Further ongoing research is needed to elucidate the safety concerns regarding SNEDDS and modification of the formulation to guarantee their future application.

\section{Conclusion}

We had developed SNEDDS to address the problem of low drug bioavailability after RYGB surgery. SNEDDS formed nanosized and globular droplets in gastric fluid with a moderately sized distribution. Our pharmacokinetic results indicate that SNEDDS increase the bioavailability of silibinin above that achieved by suspension and solution formulations of silymarin. This may be due to the collective mechanisms of SNEDDS, which have a larger surface area, resulting in better dissolution, enhanced permeability, and lymphatic absorption, and reduced lipid digestion by bile salts in RYGB rats. SNEDDS could adhere to the intestinal membrane, enabling easy penetration into the mucosa. Preliminary histopathology indicates that the GI mucosa can tolerate SNEDDS, although upregulation of COX-2 was found. SNEDDS delivered orally potentially allow better delivery of silymarin, and may overcome the problem of malabsorption caused by bariatric surgery.

\section{Acknowledgment}

The authors are grateful for the financial support of Chang Gung Memorial Hospital (grants CMRPG6B0031, CMRPG6B0121).

\section{Disclosure}

The authors report no conflicts of interest in this work.

\section{References}

1. Tichansky DS, Boughter JD Jr, Harper J, Glatt AR, Madan AK. Gastric bypass surgery in rats produces weight loss modeling after human gastric bypass. Obes Surg. 2008;18:1246-1250.

2. Samaras TT, Storms LH, Elrick H. Longevity, mortality and body weight. Ageing Res Rev. 2002;1:673-691. 
3. Adams TD, Gress RE, Smith SC, et al. Long-term mortality after gastric bypass surgery. N Engl J Med. 2007;357:753-761.

4. Schauer PR, Ikramuddin S, Gourash W, Ramanathan R, Luketich J. Outcomes after laparoscopic Roux-en-Y gastric bypass for morbid obesity. Ann Surg. 2000;232:515-529.

5. Shin AC, Zheng H, Townsend RL, Sigalet DL, Berthoud HR. Mealinduced hormone responses in a rat model of Roux-en-Y gastric bypass surgery. Endocrinology. 2010;151:1588-1597.

6. Miller AD, Smith KM. Medication and nutrient administration considerations after bariatric surgery. Am J Health Syst Pharm. 2006;63:1852-1857.

7. Balakumar K, Raghavan CV, Selvan NT, Prasad RH, Abdu S. Self nanoemulsifying drug delivery system (SNEDDS) of rosuvastatin calcium: design, formulation, bioavailability and pharmacokinetic evaluation. Colloids Surf B Biointerfaces. 2013;112:337-343.

8. Tran TH, Guo Y, Song D, Bruno RS, Lu X. Quercetin-containing self-nanoemulsifying drug delivery system for improving oral bioavailability. J Pharm Sci. 2014;103:840-852.

9. Stylopoulos N, Hoppin AG, Kaplan LM. Roux-en-Y gastric bypass enhances energy expenditure and extends lifespan in diet-induced obese rats. Obesity. 2009;17:1839-1847.

10. Fabbrini E, Sullivan S, Klein S. Obesity and nonalcohol fatty liver disease: biochemical, metabolic, and clinical implications. Hepatology 2010;51:679-689.

11. Cacciapuoti F, Scognamiglio A, Palumbo R, Forte R, Cacciapuoti F. Silymarin in non alcoholic fatty liver disease. World J Hepatol. 2013;5: 109-113.

12. Fehér J, Lengyel G. Silymarin in the prevention and treatment of liver diseases and primary liver cancer. Curr Pharm Biotechnol. 2012;13: 210-217.

13. Woo JS, Kim TS, Park JH, Chi SC. Formulation and biopharmaceutical evaluation of silymarin using SMEDDS. Arch Pharm Res. 2007;30: $82-89$.

14. Huang YB, Tsai MJ, Wu PC, Tsai YH, Wu YH, Fang JY. Elastic liposomes as carriers for oral delivery and the brain distribution of (+)-catechin. J Drug Target. 2011;19:709-718.

15. Gažák R, Svobodová A, Psotová J, et al. Oxidised derivatives of silybin and their antiradical and antioxidant activity. Bioorg Med Chem. 2004 12:5677-5687.

16. Hsu SH, Wen CJ, Al-Suwayeh SA, Huang YJ, Fang JY. Formulation design and evaluation of quantum dot-loaded nanostructured lipid carriers for integrating bioimaging and anticancer therapy. Nanomedicine (Lond). 2013;8:1253-1269.

17. Chen CC, Tsai TH, Huang ZR, Fang JY. Effects of lipophilic emulsifiers on the oral administration of lovastatin from nanostructured lipid carriers: physicochemical characterization and pharmacokinetics. Eur J Pharm Biopharm. 2010;74:474-482.

18. Zhu JX, Tang D, Feng L, et al. Development of self-microemulsifying drug delivery system for oral bioavailability enhancement of berberine hydrochloride. Drug Dev Ind Pharm. 2013;39:499-506.

19. Tsai MJ, Huang YB, Wu PC, et al. Oral apomorphine delivery from solid lipid nanoparticles with different monostearate emulsifiers: pharmacokinetic and behavioral evaluations. J Pharm Sci. 2011;100:547-557.

20. Quinn R. Comparing rat's to human's age: how old is my rat in people years? Nutrition. 2005;21:775-777.

21. Sjöström L, Narbro K, Sjöström CD, et al. Effects of bariatric surgery on mortality in Swedish obese subjects. N Engl J Med. 2007;357: 741-752.

22. Shin AC, Zheng H, Townsend RL, Patterson LM, Holmes GM, Berthoud HR. Longitudinal assessment of food intake, fecal energy loss, and energy expenditure after Roux-en-Y gastric bypass surgery in high-fat-fed obese rats. Obes Surg. 2013;23:531-540.

23. Meguid MM, Ramos EFB, Suzuki S, et al. A surgical rat model of human Roux-en-Y gastric bypass. J Gastrointest Surg. 2004;8:621-630.

24. Leighton JA, Bay MK, Maldonado AL, Johnson RF, Schenker S, Speeg KV. The effect of liver dysfunction on colchicines pharmacokinetics in the rat. Hepatology. 1990;11:210-215.
25. Holm R, Tønsberg H, Jørgensen EB, Abedinpour P, Farsad S, Müllertz A. Influence of bile on the absorption of halofantrine from lipid-based formulations. Eur J Pharm Biopharm. 2012;81:281-287.

26. Guijarro A, Suzuki S, Chen C, et al. Characterization of weight loss and weight regain mechanisms after Roux-en-Y gastric bypass in rats Am J Physiol Regul Integr Comp Physiol. 2007;293:1474-1489.

27. Stemmer K, Bielohuby M, Grayson BE, et al. Roux-en-Y gastric bypass surgery but not vertical sleeve gastrectomy decreases bone mass in male rats. Endocrinology. 2013;154:2015-2024.

28. Wu JW, Lin LC, Tsai TH. Drug-drug interactions of silymarin on the perspective of pharmacokinetics. J Ethnopharmacol. 2009;121:185-193.

29. Smith A, Henriksen B, Cohen A. Pharmacokinetic considerations in Roux-en-Y gastric bypass patients. Am J Health Syst Pharm. 2011;68: 2241-2247.

30. Thomas N, Holm R, Müllertz A, Rades T. In vitro and in vivo performance of novel supersaturated self-nanoemulsifying drug delivery systems (super-SNEDDS). J Control Release. 2012;160:25-32.

31. Baek MK, Lee JH, Cho YH, Kim HH, Lee GW. Self-microemulsifying drug-delivery system for improved oral bioavailability of pranlukast hemihydrates: preparation and evaluation. Int J Nanomedicine. 2013;8:167-176.

32. Rege BD, Kao J, Pollia J. Effects of nonionic surfactants on membrane transporters in Caco-2 cell monolayers. Eur J Pharm Sci. 2002;16:237-246.

33. Lundin P, Bojrup M, Ljusberg-Wahren H, Westrom B, Lundin S. Enhancing effects of monohexanoin and two other medium-chain glyceride vehicles on intestinal absorption of desmopressin (dDAVP) J Pharmacol Exp Ther. 1997;282:585-590.

34. von Suesskind-Schwendi M, Gruber M, Touraud D, et al. Pharmacokinetics of a self-microemulsifying drug delivery system of tacrolimus. Biomed Pharmacother. 2013;67:469-473.

35. Schrieber SJ, Wen Z, Vourvahis M, et al. The pharmacokinetics of silymarin is altered in patients with hepatitis $\mathrm{C}$ virus and nonalcoholic fatty liver disease and correlates with plasma caspase-3/7 activity. Drug Metab Dispos. 2008;36:1909-1916.

36. Javed S, Kohli K, Ali M. Reassessing bioavailability of silymarin Altern Med Rev. 2011;16:239-249.

37. Brantley SJ, Oberlies NH, Kroll DJ, Paine MF. Two flavonolignans from milk thistle (Silybum maranum) inhibit CYP2C9-mediated warfarin metabolism at clinically achievable concentrations. J Pharmacol Exp Ther. 2010;332:1081-1087.

38. MekjaruskulC, Yang YT, LeedMG, Sadgrove MP, Jay M, SripanidkulchaiB. Novel formulation strategies for enhancing oral delivery of methoxyflavones in Kaempferia parviflora by SMEDDS or complexation with 2-hydroxypropyl- $\beta$-cyclodextrin. Int J Pharm. 2013;445:1-11.

39. Bandyopadhyay S, Katare OP, Singh B. Optimized self nano-emulsifying systems of ezetimibe with enhanced bioavailability potential using long chain and medium chain triglycerides. Colloids Surf B Biointerfaces. 2012;100:50-61.

40. Larsen AT, Ohlsson AG, Polentarutti B, et al. Oral bioavailability of cinnarizine in dogs: relation to SNEDDS droplet size, drug solubility and in vitro precipitation. Eur J Pharm Sci. 2013;48:339-350.

41. Rao SV, Agarwal P, Shao J. Self-nanoemulsifying drug delivery systems (SNEDDS) for oral delivery of protein drugs II. In vitro transport study. Int J Pharm. 2008;362:10-15

42. Bernkop-Schnürch A. Nanocarrier systems for oral drug delivery: do we really need them? Eur J Pharm Sci. 2013;49:272-277.

43. Müller RH, Runge S, Ravelli V, Mehnert W, Thünemann AF, Souto EB. Oral bioavailability of cyclosporine: solid lipid nanoparticles $\left(\mathrm{SLN}^{\circledR}\right)$ versus drug nanocrystals. Int J Pharm. 2006;317:82-89.

44. Ponsky TA, Brody F, Pucci E. Alterations in gastrointestinal physiology after Roux-en-Y gastric bypass. J Am Coll Surg. 2005;201:125-131.

45. Zhao G, Huang J, Xue K, Si L, Li G. Enhanced intestinal absorption of etoposide by self-microemulsifying drug delivery systems: roles of P-glycoprotein and cytochrome P450 3A inhibition. Eur J Pharm Sci. 2013;50:429-439.

46. Nielsen FS, Petersen KB, Müllertz A. Bioavailability of probucol from lipid and surfactant based formulations in minipigs: influence of droplet size and dietary state. Eur J Pharm Biopharm. 2008;69:553-562. 
47. Weerapol Y, Limmatvapirat S, Nunthanid J, Sriamornsak P. Selfnanoemulsifying drug delivery system of nifedipine: impact of hydrophiliclipophilic balance and molecular structure of mixed surfactants. AAPS Pharm Sci Tech. 2014;15:456-464.

48. Heshmati N, Cheng X, Eisenbrand G, Fricker G. Enhancement of oral bioavailability of E804 by self-nanoemulsifying drug delivery system (SNEDDS) in rats. J Pharm Sci. 2013;102:3792-3799.
49. Gažák R, Walterová D, Křen V. Silybin and silymarin - new and emerging applications in medicine. Curr Med Chem. 2007;14:315-338.

50. Crofford LJ. COX-1 and COX-2 tissue expression: implications and predictions. J Rheumatol Suppl. 1997;49:15-19.

51. Kiss L, Walter FR, Bocsik A, et al. Kinetic analysis of the toxicity of pharmaceutical excipients Cremophor EL and RH40 on endothelial and epithelial cells. J Pharm Sci. 2013;102:1173-1181.

\section{Publish your work in this journal}

The International Journal of Nanomedicine is an international, peerreviewed journal focusing on the application of nanotechnology in diagnostics, therapeutics, and drug delivery systems throughout the biomedical field. This journal is indexed on PubMed Central, MedLine, CAS, SciSearch ${ }^{\circledR}$, Current Contents ${ }^{\circledR} /$ Clinical Medicine,
Journal Citation Reports/Science Edition, EMBase, Scopus and the Elsevier Bibliographic databases. The manuscript management system is completely online and includes a very quick and fair peer-review system, which is all easy to use. Visit http://www.dovepress.com/ testimonials.php to read real quotes from published authors. 